NASA Technical Memorandum 105349

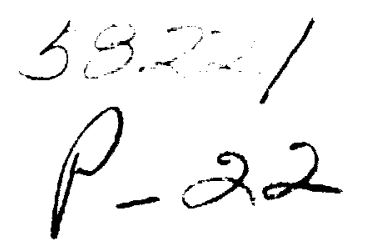

\title{
On the Thermodynamic Framework of Generalized Coupled Thermoelastic- Viscoplastic-Damage Modeling
}

S.M. Arnold

Lewis Research Center

Cleveland, Ohio

and

A.F. Saleeb

University of Akron

Akron, Ohio

\section{N/SA}

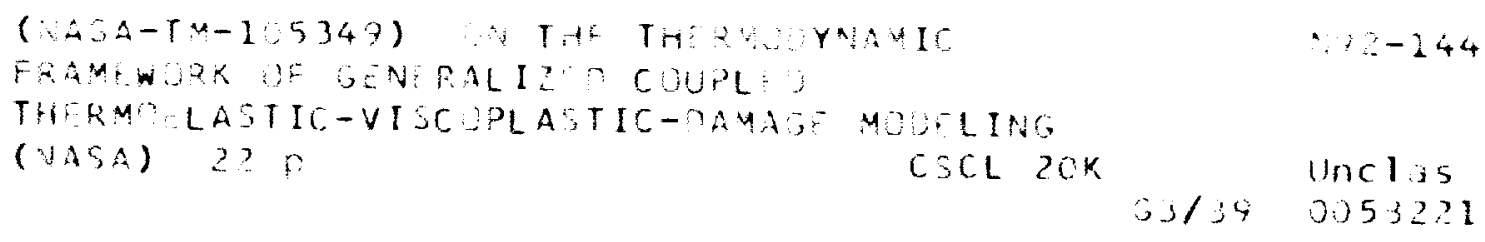




\title{
ON THE THERMODYNAMIC FRAMEWORK OF GENERALIZED COUPLED THERMOELASTIC-VISCOPLASTIC-DAMAGE MODELING
}

\author{
S.M. Arnold \\ National Aeronautics and Space Administration \\ Lewis Research Center \\ Cleveland, Ohio 44135
}

and

\author{
A.F. Saleeb \\ University of Akron \\ Department of Civil Engineering \\ Akron, Ohio 44325
}

\begin{abstract}
A complete potential based framework utilizing internal state variables is put forth for the derivation of reversible and irreversible constitutive equations. In this framework the existence of the total (integrated) form of either the (Helmholtz) free energy or the (Gibbs) complementary free energy are assumed a priori. Two options for describing the flow and evolutionary equations are described, wherein option one (the fully coupled form) is shown to be over restrictive while the second option (the decoupled form) provides significant flexibility. As a consequence of the decoupled form a new operator, i.e. the Compliance operator, is defined which provides a link between the assumed Gibb's and complementary dissipation potential and ensures a number of desirable numerical features, for example the symmetry of the resulting consistent tangent stiffness matrix. An important conclusion reached, is that although many theories in the literature do not conform to the general potential framework outlined, it is still possible in some cases, by slight modifications of the employed forms, to restore the complete potential structure.
\end{abstract}

\subsection{INTRODUCTION}

In general, the inelasticity exhibited by the thermomechanical response of engineering materials is related to irreversible thermodynamic processes. These involve energy dissipations and material stiffness variations due to physical changes in the microstructure. Consequently, thermodynamic arguments have often been utilized as a foundation on which phenomenological constitutive laws may be formulated, i.e., the so-called internal variable formalism [1-8]. Material descriptions such as elastoplastic, viscoplastic, continuum-based damage, etc., (all falling into this framework) have been subjects of extensive research over the years [8-21].

From a strict mathematical standpoint, however, the "thermodynamic admissibility" restriction associated with the dissipative mechanisms underlying the above material models reduce to the well-known (local) Clausius-Duhem or dissipation inequality $[1,2]$. Indeed, this is the form that is typically employed in discussing the general structure of the "thermodynamically -based" constitutive equations developed $[1-5,8,13]$; whereby the mathematical constructs such as the flow or dissipation potential and the attendant normality/generalized normality relations, i.e., the $\Omega$ - form $[6,8,9,15-17,20,22]$ 
are introduced solely for convenience in satisfying the above constraints based on simple properties of non-negativeness and convexity of these functions. We emphasize, however, that such forms do not presuppose, or automatically imply, the existence of the total (integrated) forms of the associated thermodynamic potentials ; e.g. the (Helmholtz) free energy, or the (Gibbs) complementary free energy. When the latter are utilized a priori, the corresponding formulations will be described as exhibiting a complete potential - based structure; on the other hand, those derived from an assumed $\Omega$-form are referred to as incomplete potential-based models.

The (complete) potential-based class of inelastic constitutive equations possess a number of distinct and important attributes, from both a theoretical as well as computational standpoint. First, they constitute the cornerstone of numerous regularity properties and bounding (or limit) theorems in plasticity and viscoplasticity [22-26].

Secondly, they result in a sufficiently general variational structure, whose properties can be exploited to derive a number of useful material conservation laws [27-29]. Thirdly, on the numerical side, the discrete form of this same variational structure is of great advantage in the development of efficient algorithms for finite element implementation; e.g.,

symmetry-preserving material tangent stiffness operators are easily obtained in implicit solution schemes [30-35]. Finally, this complete potential based framework conveniently lends itself to intelligent application of symbolic manipulation systems which facilitate the construction, implementation and analysis of new deformation and damage models [36,37].

With the above background in mind, our objective is to study the general form, and the ensuing restrictions imposed by a complete potential-based viscoplastic formulation, in terms of the Gibb's thermodynamic potential, particularly in regard to several of the presently used forms of the flow and evolutionary laws. It appears that although many of these theories [e.g. 5,6,10,15-18] do not conform to the general potential framework outlined, it is still possible in some cases, by slight modifications of the employed forms, to restore the complete potential structure. However, the question still remains as to whether these modifications will significantly affect the predictive capabilities achieved by the original forms. This is not addressed here, but will be addressed in a subsequent paper $[38]$.

\subsection{THEORETICAL FRAMEWORK}

We begin by summarizing the basic thermodynamic equations governing the thermo-mechanical behavior of an initially isotropic material element. An assumed expression for the Gibb's thermodynamic potential function [1-3] in terms of a number of state and internal variables characterizing the changing internal structure of the material, 
is taken. For conciseness, the discussion is limited to the case of small deformations, in which the initial state is assumed to be stress free throughout. Also a cartesian reference frame and index notation (with summation implied on repeated roman subscripts) is utilized. It is noticed that all subsequent equations and discussions can be equivalently rephrased, using appropriate Legendre transformations, in terms of the (Helmholtz) free energy and the associated complementary variables.

In its volumetric differential form, the Gibb's thermodynamic potential $\mathrm{G}\left(\sigma_{\mathrm{ij}}, \alpha_{\beta}, \mathrm{T}\right)$, can be written as follows $[2,25]$ :

$$
\mathrm{dG}=-_{\mathrm{ij}} \mathrm{d} \sigma_{\mathrm{ij}}-\mathrm{SdT}-\mathrm{A}_{\beta} \mathrm{d} \alpha_{\beta}
$$

where $\mathrm{S}$ denotes the entropy, $\alpha_{\beta}$ the internal state variables, $\mathrm{A}_{\beta}$ the thermodynamic affinities corresponding to $\alpha_{\beta}$ (where $\beta=1, . . N$ and the tensorial character of these internal variables will be defined in the particular context), $\mathrm{e}_{\mathrm{ij}}$ the total strain and $\sigma_{\mathrm{ij}}$ the cauchy stress tensor. It follows from equation (1) then that

$$
\begin{array}{ll}
e_{i j}=\frac{-\partial G}{\partial \sigma_{i j}} & \text { (total strain) } \\
S=\frac{-\partial G}{\partial T} & \text { (entropy) }
\end{array}
$$

and

$$
A_{\beta}=\frac{-\partial G}{\partial \alpha_{\beta}} \quad \text { (affinity) }
$$

are defined as the equations of state $[8,39]$ and $\sigma_{\mathrm{ij}}$, T, and $\alpha_{\beta}$ are the "force-like" thermodynamic state variables while $e_{i j}, S$, and $A_{\beta}$ are the conjugate "displacement-like" variables.

Now considering the following general form for the Gibb's potential:

$$
\mathrm{G}=\mathrm{G}\left(\sigma_{\mathrm{ij}}, \alpha_{\beta}, \mathrm{T}\right)
$$

where initially no restrictions are imposed on the functional dependence other than as declared above. The most general expression for the total strain rate can then be obtained 
by differentiating equation (2). That is

$$
\dot{\mathrm{e}}_{\mathrm{ij}}=\frac{\mathrm{d}}{\mathrm{dt}}\left(\frac{-\partial \mathrm{G}}{\partial \sigma_{\mathrm{ij}}}\right)=\frac{-\partial^{2} \mathrm{G}}{\partial \sigma_{\mathrm{i} j} \partial \sigma_{\mathrm{rs}}} \dot{\sigma}_{\mathrm{rs}}+\frac{-\partial^{2} \mathrm{G}}{\partial \sigma_{\mathrm{i} j} \partial \alpha_{\beta}} \dot{\alpha}_{\beta}+\frac{-\partial^{2} \mathrm{G}}{\partial \sigma_{\mathrm{i} j} \partial \mathrm{T}} \dot{\mathrm{T}}
$$

With the above in mind, two options are actually available for describing the flow and evolutionary equations. The first option assumes a fully coupled form, i.e., one in which the irreversible strain rate is intimately linked to the thermodynamic internal state, hence its functional dependence (evolution) is completely defined once $G$ is assumed. The second option is a decoupled Gibb's form, in which the selected internal state variables are grouped a priori by separation of the inelastic strain as an independent state parameter and suppressing all stress dependency of the remaining associated internal state groups in the selected $\mathrm{G}$ function. Both of these options are described separately, below.

\subsection{Fully Coupled Form}

Consider the following coupled form for the Gibb's potential:

$$
\mathrm{G}=\mathrm{E}\left(\sigma_{\mathrm{ij}}\right)+\mathrm{M}\left(\sigma_{\mathrm{ij}}, \alpha_{\beta}\right)+\mathrm{H}\left(\alpha_{\beta}\right)-\mathrm{Z}(\mathrm{T})-\frac{\sigma_{\mathrm{k} \mathrm{k}}}{3} \gamma\left(\mathrm{T}-\mathrm{T}_{0}\right)
$$

where initially certain restrictions (coming from the affinities $\mathrm{A}_{\beta}$, discussed subsequently) are imposed on the functions $E$ and $M, T_{0}$ is a reference temperature and $\gamma$ is the volumetric coefficient of thermal expansion. Substitution of equations (4) and (6) into (5) yields a particular expression for the total strain rate, i.e.,

$$
\dot{\mathrm{e}}_{\mathrm{ij}}=\left[\frac{-\partial^{2} \mathrm{E}}{\partial \sigma_{\mathrm{ij}} \partial \sigma_{\mathrm{rs}}}+\frac{-\partial^{2} \mathrm{M}}{\partial \sigma_{\mathrm{i} j}}{ }_{\mathrm{j} \sigma_{\mathrm{rs}}}\right] \dot{\sigma}_{\mathrm{rs}}+\left[\frac{\partial \mathrm{A}_{\beta}}{\partial \sigma_{\mathrm{i} j}}\right] \dot{\alpha}_{\beta}+\delta_{\mathrm{ij}} \gamma \dot{\mathrm{T}}
$$

in which the above three terms may be identified as the reversible, irreversible and thermal expansion components of the total strain rate, respectively. Thus,

$$
\dot{e}_{i j}=\dot{\epsilon}_{i j}^{R}+\dot{\epsilon}_{i j}^{I}+\dot{\epsilon}_{i j}^{T}
$$


with

$$
\begin{aligned}
& \dot{\epsilon}_{\mathrm{ij}}^{\mathrm{R}}=\left[\frac{-\partial^{2} \mathrm{E}}{\partial \sigma_{\mathrm{i} j} \partial \sigma_{\mathrm{rs}}}+\frac{-\partial^{2} \mathrm{M}}{\partial \sigma_{\mathrm{i}}} \frac{\mathrm{j}_{\mathrm{rs}}}{\partial \sigma_{\mathrm{rs}}}\right. \\
& \dot{\epsilon}_{\mathrm{ij}}^{\mathrm{I}}=\left[\frac{\left.\partial \mathrm{A}_{\beta}\right] \dot{\alpha}_{\beta}}{\partial \sigma_{\mathrm{ij}}} \quad\right. \text { (reversible) } \\
& \dot{\epsilon}_{\mathrm{ij}}^{\mathrm{T}}=\delta_{\mathrm{ij}} \gamma \dot{\mathrm{T}}
\end{aligned}
$$

Furthermore expressions for the entropy and "displacement-like" affinities can be obtained from equations (3), (4), and (6), i.e.,

$$
\mathrm{S}=\frac{\partial \mathrm{Z}}{\partial \mathrm{T}}+\frac{\sigma_{\mathbf{k k}}}{3} \gamma
$$

and

$$
\mathrm{A}_{\beta}=\frac{-\partial \mathrm{M}}{\partial \bar{\alpha}_{\beta}}-\frac{\partial \mathrm{H}}{\partial \alpha_{\beta}}
$$

thus implying the functional dependence of $S=S\left(T, \sigma_{k k}\right)$ and $A_{\beta}=A_{\beta}\left(\sigma_{\text {rs }}, \alpha_{\eta}\right)$.

Consequently, in its present coupled form certain restrictions now apply to the functions $M$ and $H$ in order for $A_{\beta}$ to have the above functional dependence. For instance, $M$ must always be at least linear in stress, and furthermore, given an $H$ that is at least quadratic in internal state will require that $M$ be at least linear in internal state $\alpha_{\beta}$; alternatively given an $H$ that is assumed to be linear (or zero) in internal state requires that $M$ be at least quadratic in internal state.

Now assuming the existence of a dissipation potential, $\varphi\left(\mathrm{A}_{\beta}, \mathrm{S}\right)$, the rate of change of the internal state variables $\left(\alpha_{\beta}\right)$ can be expressed in terms of their corresponding affinities. That is, the evolution or kinetic law becomes:

$$
\dot{\alpha}_{\ell}=\frac{\partial \varphi}{\partial \mathrm{A}_{\ell}}
$$

Furthermore, considering equations (12) and (13), it is clear that a correspondence between $\varphi$ and a complementary dissipation potential $\Omega=\Omega\left(\sigma_{\mathrm{ij}}, \alpha_{\beta}, \mathrm{T}\right)$ exist, since $\varphi$ is an implicit 
function of $\sigma_{\mathrm{ij}}$ and $\alpha_{\beta}$ through $\mathrm{A}_{\beta}$, i.e., $\varphi=\varphi\left(\mathrm{A}_{\beta}\left(\sigma_{\mathrm{ij}} \alpha_{\beta}\right), \mathrm{T}\right)$. Note defining the dependence of $\Omega$ on $\sigma_{\mathrm{ij}}, \alpha$ and $\mathrm{T}$ does not preclude the inclusion (or dependence) of the associated thermodynamic affinities as parameters (as opposed to "true" variables), e.g. $\mathrm{A}_{\beta}$ or $\epsilon$ [cf. $5,8]$.

Here, in the fully coupled option, we make use of equations (10), (13) and (14) directly to obtain expressions for the flow, i.e.,

$$
\dot{\epsilon}_{\mathrm{ij}}^{\mathrm{I}}=\left[\frac{\partial \mathrm{A}}{\partial \sigma_{\mathrm{ij}}}\right] \dot{\alpha}_{\beta}=\frac{\partial \varphi}{\partial \sigma_{\mathrm{ij}}}
$$

and evolutionary laws, cf. equation (14),

$$
\dot{\alpha}_{\beta}=\frac{\partial \varphi}{\partial \mathrm{A}_{\beta}}
$$

where $A_{\beta}$ is obtained from equation (13). Note that $A_{\beta}$ and thus the functional dependence of $\varphi$ is completely defined once the functions $M$ and $H$ are assumed.

It is evident that the present form is too restrictive (see Onat and Leckie [6] for a number of examples), in that it requires proportionality between the inelastic strain and the rate of change of internal state to maintain consistency between the two expressions for inelastic strain rates in equation (10) and (15).

\subsection{Decoupled Form}

Here, a less restrictive, yet equivalent, decoupled form can be derived by assuming a form a priori for the Gibb's potential whereby the inelastic strain is assumed to be an independent parameter (and not an internal state variable) with two other groupings of internal "force-like" state variables associated with irreversibility, i.e., $\alpha_{\beta}$ which are associated with the material inelasticity and $\mathrm{D}_{\beta}$ which are associated with the degradation of the material due to some damage mechanisms $[8,12,13]$. As an example, one may write

$$
\mathrm{G}=\mathrm{E}\left(\sigma_{\mathrm{ij}}\right)+\sigma_{\mathrm{ij}} \epsilon_{\mathrm{ij}}^{\mathrm{I}}+\mathrm{M}\left(\sigma_{\mathrm{ij}}, \mathrm{D} \beta\right)+\mathrm{H}\left(\alpha_{\beta}\right)-\mathrm{Z}(\mathrm{T})-\frac{\sigma_{\mathrm{k} \mathrm{k}}}{3}\left(\mathrm{~T}-\mathrm{T}_{\mathrm{o}}\right)
$$

Where, once again, differentiating equation (2) with the above assumed form for $G$, an expression defining the total strain rate can be obtained, i.e., 


$$
\dot{\mathrm{e}}_{\mathrm{ij}}=\left[\frac{-\partial^{2} \mathrm{E}}{\partial \sigma_{\mathrm{ij}} \partial \sigma_{\mathrm{rs}}}+\frac{-\partial^{2} \mathrm{M}}{\partial \sigma_{\mathrm{i} j}}\right] \dot{\sigma}_{\mathrm{rs}}-\dot{\epsilon}_{\mathrm{ij}}^{\mathrm{I}}+\left[\frac{\partial \Lambda_{\beta}}{\partial \sigma_{\mathrm{ij}}}\right] \dot{\mathrm{D}}_{\beta}+\delta_{\mathrm{ij}} \gamma \dot{\mathrm{T}}
$$

in which the above four terms may be identified as the reversible, inelastic, irreversible and thermal expansion components of the total strain rate, respectively. Note that $\Lambda_{\beta}$ are the "displacement-like" thermodynamic affinities associated with the internal material degradation "force-like" variables $\mathrm{D}_{\beta}$. Thus,

$$
\dot{\mathrm{e}}_{\mathrm{ij}}=\dot{\epsilon}_{\mathrm{ij}}^{\mathrm{R}}+\dot{\epsilon}_{\mathrm{ij}}^{\mathrm{I}}+\dot{\epsilon}_{\mathrm{ij}}^{\mathrm{D}}+\dot{\epsilon}_{\mathrm{ij}}^{\mathrm{T}}
$$

with

$$
\begin{array}{ccc}
\dot{\epsilon}_{\mathrm{ij}}^{\mathrm{R}}=\left[\frac{\partial^{2} \mathrm{E}}{\partial \sigma_{\mathrm{i} \mathrm{j}} \partial \sigma_{\mathrm{rs}}}+\frac{-\partial^{2} \mathrm{M}}{\partial \sigma_{\mathrm{i}}} \frac{\dot{\mathrm{j}}_{\mathrm{rs}}}{\partial \sigma_{\mathrm{rs}}}\right. & \text { (reversible) } \\
\dot{\epsilon}_{\mathrm{ij}}^{\mathrm{D}}=\left[\frac{\left.\partial \Lambda_{\beta}\right] \dot{\mathrm{D}}_{\beta}}{\partial \sigma_{\mathrm{ij}}}\right. & \text { (irreversible) } \\
\dot{\epsilon}_{\mathrm{i} \mathrm{j}}^{\mathrm{T}}=\delta_{\mathrm{ij}} \gamma \dot{\mathrm{T}} & \text { (thermal) }
\end{array}
$$

and $\dot{\epsilon}_{\mathrm{ij}}$ (inelastic strain rate) is to be defined separately, subsequently. Note also that the definition of $\Lambda_{\beta}$ follows that of equation (13) (with the suppression of $H$ and equivalance of $\alpha_{\beta}$ and $D_{\beta}$ ) and the evolution of $\mathrm{D}$, that of equation (14) given an assumed form of a damage dissipation potential $\Psi=\Psi\left(\Lambda_{\beta}\right)$. Various coupled and decoupled deformation and damage formulations may thus be investigated using this framework.

To provide a framework for the definition of the inelastic strain rate $\left(\dot{\epsilon}_{\mathrm{ij}}^{\mathrm{I}}\right)$ and ensure the thermodynamic admissibility of it, the requirement that the Clausius- Duhem or dissipation inequality be satisfied is introduced. That is,

$$
\mathrm{d} \Omega\left(\sigma_{\mathrm{ij}}, \alpha_{\beta}\right)=\sigma_{\mathrm{ij}} \dot{\epsilon}_{\mathrm{ij}}^{\mathrm{I}}-\dot{\mathrm{A}}_{\beta} \alpha_{\beta} \geq 0
$$

thus differentiating once with respect to stress $\left(\sigma_{\mathrm{ij}}\right)$ and once with respect to internal state, gives: 


$$
\dot{\epsilon}_{\mathrm{ij}}^{\mathrm{I}}=\frac{\partial \Omega}{\partial \sigma_{\mathrm{ij}}}
$$

and

$$
\dot{\mathrm{A}}_{\beta}=\frac{-\partial \Omega}{\partial \alpha_{\beta}}
$$

Returning to equation (4) and differentiating with respect to time, an expression for the rate of change of the affinities $A_{\beta}$ in terms of the rate of change of the corresponding internal state can be obtained, i.e.,

$$
\begin{aligned}
& \dot{\mathrm{A}}_{\beta}=\frac{\mathrm{d}}{\mathrm{dt}}\left[\frac{-\partial \mathrm{G}}{\partial \alpha_{\beta}}\right]=-\frac{\partial^{2} \mathrm{H}}{\partial \alpha_{\ell} \partial \alpha_{\beta}} \dot{\alpha}_{\ell} \\
& \dot{\mathrm{A}}_{\beta}=\left[\mathrm{Q}_{\beta \ell}\right] \dot{\alpha}_{\ell}
\end{aligned}
$$

where

$$
\mathrm{Q}_{\beta \ell}=\frac{-\partial^{2} \mathrm{H}}{\partial \alpha_{\beta} \partial \alpha_{\ell}}
$$

given the assumed form of the Gibb's potential in equation (16). Due to the fact that the operator $Q$ relates the "force-like" state variables to the "displacement-like" variables and is completely defined once a Gibb's potential is chosen, this operator will be defined as the Compliance operator henceforth. Furthermore it is interesting to note that this operator provides information relative to the curvature of the Gibb's potential as well as the relaxation trajectories in the associated state space [40].

Now substituting equation (25) into (24) and rearranging terms, gives,

$$
\dot{\alpha}_{\ell}=-\left[\mathrm{Q}_{\beta \ell}^{-1}\right] \frac{\partial \Omega}{\partial \alpha_{\beta}}
$$

which defines the evolution of internal state. Thus equations (23) and (27) represent the flow and evolutionary laws, respectively, for an assumed $\Omega=\Omega\left(\sigma_{\mathrm{ij}}, \alpha_{\beta}, \mathrm{T}\right)$ and Gibb's potential wherein both potentials are directly linked through the internal state variables $\alpha_{\beta}$. It is interesting to note that only under special conditions (e.g., uniaxial states of stress and scalar internal state variables) does equation (27) reduce to those discussed by Ponter and Ponter and Leckie $[20,22,25]$. 
Clearly, this framework provides a structure in which the flow and evolution laws are associative, satisfying equation (26) explicitly, and are fully integrable . Furthermore, this option provides significant freedom (i.e., $d \Omega \geq 0$ ) in the definition of the complementary dissipation potential so that various complete potential based formulations can be expressed. The price for this additional freedom, however, is in general the non-trivial inversion of the compliance operator.

\subsection{DISCUSSION OF SPECIAL CASES}

Equations (14) and (15), and, (23) and (27), provide the general thermodynamic framework whereby most, if not all, potential based models with associated flow and evolutionary laws can be derived. To illustrate this a number of classical theories as well as a few nonclassical forms will be recovered, depending of course upon the assumed form for the Gibb's potential, i.e., functions $\mathrm{E}, \mathrm{M}$, and $\mathrm{H}$.

\subsection{Elasticity}

Here, if $\mathbf{E}$ is assumed to be quadratic in stress, i.e.,

$$
\mathrm{E}=-\frac{1}{2} \mathrm{C}_{\mathrm{rskl}} \sigma_{\mathrm{rs}} \sigma_{\mathrm{kl}}
$$

and $M$ is assumed to be a separable function linear in stress and internal state (where, $\alpha_{\beta}$ is taken as a symmetric second order tensor),

$$
\begin{aligned}
& \mathrm{M}=\mathrm{L}\left(\sigma_{\mathrm{rs}}\right) \mathrm{P}\left(\alpha_{\beta}\right) \\
& =\sigma_{\mathrm{rs}} \alpha_{\mathrm{rs}}
\end{aligned}
$$

and $\mathrm{H}$ is taken as zero, then from equation (9);

$$
\epsilon_{\mathrm{ij}}^{\mathrm{R}}=\mathrm{C}_{\mathrm{ijrs}} \sigma_{\mathrm{rs}}
$$

the classical form describing generalized Hooke's Law [41] is obtained. If an expression describing nonlinear elastic behavior is desired any scalar form linear or nonlinear in stress for $\mathrm{E}$ and/or $\mathrm{M}$ can be chosen. 


\subsection{Coupled Damage Elasticity}

If $\alpha_{\beta}$ is associated with material degradation, i.e., "damage", classical forms of an elastically damaging material can be recovered. Here, $\mathbf{E}$ is assumed as before to be quadratic in stress (see equation (28)) while $\mathrm{M}$ and $\mathrm{H}$ are taken as follows:

$$
\mathrm{M}=\frac{-1}{2} \mathscr{D C} \mathrm{rskl}_{\mathrm{rs}} \sigma_{\mathrm{kl}}
$$

and

$$
\mathrm{H}=\mathrm{h} \mathscr{D}^{\mathrm{n}}
$$

where the internal state variable $\left(\alpha_{\beta} \equiv \mathscr{D}\right)$ is the scalar "force-like" damage affinity.

Now calculating the total strain rate, as given in equation (7), we find

$$
\dot{\mathrm{e}}_{\mathrm{ij}}=[1-\mathscr{D}] \mathrm{C}_{\mathrm{ijrs}} \dot{\sigma}_{\mathrm{rs}}-\mathrm{C}_{\mathrm{ijrs}} \sigma_{\mathrm{rs}} \dot{D}+\delta_{\mathrm{ij}} \gamma \dot{\mathrm{T}}
$$

wherein the term [1- $9 \mathrm{C}_{\mathrm{ijrs}}$ is the classical effective compliance tensor [8], and $\mathrm{C}_{\mathrm{ijrs}} \sigma_{\mathrm{rs}} \mathscr{D}$ is the irreversible strain measure. Note that the "displacement-like" affinity (equation (13)) is

$$
\mathrm{D}=\frac{1}{2} \mathrm{C}_{\mathrm{ijkl}} \sigma_{\mathrm{ij}} \sigma_{\mathrm{kl}}-\mathrm{h} \mathrm{n} \mathscr{D}^{\mathrm{n}-1}
$$

and that if $n=1$, we see that the corresponding affinity is the elastic strain energy. Now with regard to the evolution of $\mathscr{D}$ equation (14) gives,

$$
\mathscr{D}=\frac{\partial \Psi(\mathrm{D})}{\partial \mathrm{D}}
$$

\subsection{Viscoplasticity}

In order to illustrate the potential of this framework for both the coupled and decoupled option, two specific, yet general, $J_{2}$ (second invariant of the deviatoric stress) forms of the Gibb's potential shall be assumed. The only difference between these two sets is in the assumed dependence of the $M\left(\sigma_{i j}, \alpha_{\beta}\right)$ function, where in case $I, M$ is assumed separable and in case II, non-separable. Thus, 
with

$$
\begin{aligned}
& \mathrm{E}=-\frac{1}{2} \mathrm{C}_{\mathrm{rskl}} \sigma_{\mathrm{rs}} \sigma_{\mathrm{kl}} \\
& \mathrm{H}=-\mathrm{A} \oiint_{2}^{\mathrm{m}}
\end{aligned}
$$

$$
\mathscr{\not}=1 / 2 \alpha_{\mathrm{ij}} \alpha_{\mathrm{ij}}
$$

and in Case I

and in Case II

$$
\begin{aligned}
\mathrm{M} & =\mathrm{L}\left(\mathrm{S}_{\mathrm{rs}}\right) \mathrm{P}\left(\alpha_{\beta}\right) \\
& =-\mathrm{BS}_{\mathrm{rs}} \alpha_{\mathrm{rs}} \not 2
\end{aligned}
$$

with

$$
\mathrm{M}=\mathrm{B} \mathrm{J}_{2}^{\mathrm{v}} \not \mathbf{z}
$$

where

$$
\mathrm{J}_{2}=1 / 2 \Sigma_{\mathrm{ij}} \Sigma_{\mathrm{ij}}
$$

$$
\begin{aligned}
& \Sigma_{i j}=S_{i j}-\alpha_{i j} \\
& S_{i j}=\sigma_{i j}-\frac{1}{3} \sigma_{k k} \delta_{i j}
\end{aligned}
$$

Note that in the above assumed forms for $\mathrm{M}$ and $\mathrm{H}$, the internal state variable $\left(\alpha_{\beta}\right)$ is taken as a second-order and traceless tensor, and is typically identified as the back stress tensor and associated with kinematic like hardening $[6,10,15]$.

\subsubsection{Coupled Form}

Considering the coupled form, and the above definitions, the affinity $A_{i j}$ takes on the form, in:

\section{Case I}

where

$$
A_{i j}=c\left\{S_{i j}-b \alpha_{i j}\right\}
$$

and

$$
\text { c }=\mathrm{B}
$$

$$
\mathrm{b}=\frac{-\mathrm{Am} \mathbb{m}_{2}^{\mathrm{m}-1}}{\mathrm{~B} \xi_{2}^{\mathrm{n}}}-\frac{\mathrm{n}}{\mathscr{f}_{2}} \mathrm{~S}_{\mathrm{rs}} \alpha_{\mathrm{rs}} \text {. }
$$


and in,

\section{Case II}

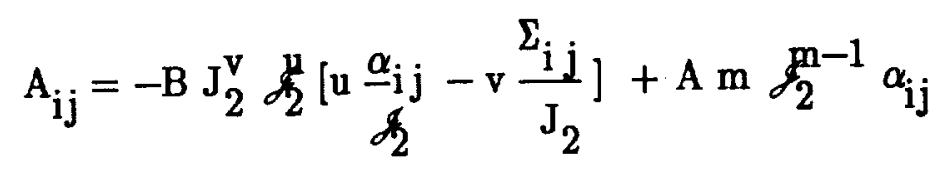

while the flow and evolutionary laws come directly from equations (15) and (14), respectively, given an assumed dissipation potential $\varphi$. It is interesting to note that in case I the typical effective stress definition found in the literature is recovered (within a factor c) when $\mathrm{n}=0, \mathrm{~m}=1$, and $\mathrm{A}=\mathrm{B}$, that is,

$$
A_{i j}=c\left\{S_{i j}-\alpha_{i j}\right\}
$$

Furthermore, note that for the above assumed forms, the Gibb's potential is a homogeneous quadratic function which is known to be convex.

For convenience, the dissipation potential $\varphi$ is assumed to have the following form;

$$
\varphi=\int \mathrm{f}\left(\mathrm{F}\left(\mathrm{A}_{\mathrm{ij}}\right)\right) \mathrm{dF}
$$

where $F$ is a scalar function, e.g., $F=A_{2} / \kappa^{2}-1$ and $A_{2}=\frac{1}{2} A_{i j} A_{i j}$, whereby it is easily shown that

$$
\begin{aligned}
& \frac{\partial \varphi}{\partial \sigma_{\mathrm{kl}}}=\mathrm{F} \Gamma_{\mathrm{rskl}} \mathrm{A}_{\mathrm{rs}} \\
& \frac{\partial \varphi}{\partial \mathrm{A}_{\mathrm{rs}}}=\mathrm{F} \mathrm{A}_{\mathrm{rs}}
\end{aligned}
$$

where

$$
F=f(F) / \kappa^{2}
$$

and for case I,

$$
\Gamma_{\text {rskl }}=\mathrm{c} \mathrm{I} I_{\text {rskl }}
$$

or in case II, 


$$
\begin{aligned}
& \Gamma_{\text {rskl }}=-B J_{2}^{\mathrm{v}} \underset{\delta}{\not}\left\{\frac{\Sigma_{\mathrm{rs}}}{\mathrm{J}_{2}}\left[\mathrm{u} \frac{\alpha_{\mathrm{kl}}}{\mathscr{C}_{2}}-\mathrm{v} \frac{\Sigma_{\mathrm{kl}}}{\mathrm{J}_{2}}\right]\right. \\
& \left.-\mathrm{v}\left[\frac{\mathrm{I}_{\mathrm{rskl}}}{\mathrm{J}_{2}}-\frac{\Sigma_{\mathrm{rs}} \Sigma_{\mathrm{kl}}}{\mathrm{J}_{2}^{2}}\right]\right\}
\end{aligned}
$$

with $\mathrm{I}_{\mathrm{rskl}}$ defined to be $\delta_{\mathrm{rk}} \delta_{\mathrm{sl}}$, such that the flow and evolutionary laws become,

$$
\begin{aligned}
& \dot{\epsilon}_{\mathrm{ij}}^{I}=F \Gamma_{r s i j} A_{r s} \\
& \dot{\alpha}_{r s}=\Gamma_{r s i j}^{-1} \dot{\epsilon}_{i j}^{I}
\end{aligned}
$$

respectively.

After examining the above flow and evolution laws one might observe that only a purely hardening material may be modeled with the above complete coupled form, since in order for steady state $\left(\dot{\alpha}_{\mathrm{rs}}=0\right)$ to occur the $\dot{\epsilon}_{\mathrm{ij}}^{\mathrm{I}}$ must be also zero. Thus no secondary creep rate could be predicted. However, as shown in the following section, if one assumes a decoupled but consistent form, a complete potential based, yet general, form can still be obtained (e.g., one with a Bailey-Orwan [20] competing mechanism in its evolution law).

\subsubsection{Decoupled Form}

Now considering a decoupled form, we can assume the complementary dissipation potential to take the form

$$
\Omega=\int \mathrm{f}\left(\mathrm{F}\left(\Sigma_{\mathrm{ij}}\right)\right) \mathrm{dF}+\int \mathrm{g}\left(\mathrm{G}\left(\alpha_{\mathrm{ij}}\right)\right) \mathrm{dG}
$$

where $F$ and $G$ are scalar functions (e.g., $F=J_{2} / \kappa^{2}-1$ and $G=\mathscr{J}_{2} / \kappa^{2}$, see [40]), thus it is easily shown that

where

$$
\begin{aligned}
& \frac{\partial \Omega}{\partial \sigma_{\mathrm{kl}}}=\mathrm{F} \Sigma_{\mathrm{kl}} \\
& \frac{\partial \Omega}{\partial \alpha_{\mathrm{ij}}}=-\left\{\frac{\partial \Omega}{\partial \sigma_{\mathrm{ij}}}-\frac{\mathrm{R}}{\mathrm{H} \kappa^{2}} \mathrm{~g}(\mathrm{G}) \alpha_{\mathrm{ij}}\right\}
\end{aligned}
$$

$$
F=f(F) / \kappa^{2}
$$


so that the flow (equation (23)) and evolutionary (equation (27)) laws become,

and

$$
\dot{\epsilon}_{\mathrm{ij}}^{\mathrm{I}}=F \Sigma_{\mathrm{ij}}
$$

$$
\begin{aligned}
\dot{\alpha}_{\mathrm{ij}} & =-\left[\mathrm{Q}_{\mathrm{rsij}}^{-1}\right] \frac{\partial \Omega}{\partial \alpha_{\mathrm{rs}}} \\
& =\left[\mathrm{Q}_{\mathrm{rsij}}^{-1}\right]\left\{\frac{\partial \Omega}{\partial \sigma_{\mathrm{rs}}}-\frac{\mathrm{R}}{\mathrm{H} \kappa^{2}} \mathrm{~g}(\mathrm{G}) \alpha_{\mathrm{rs}}\right\} \\
\dot{\alpha}_{\mathrm{ij}} & =\left[\mathrm{Q}_{\mathrm{rsij}}^{-1}\right]\left\{\dot{\epsilon}_{\mathrm{rs}}^{\mathrm{I}}-\frac{\mathrm{R}}{\mathrm{H} \kappa^{2}} \mathrm{~g}(\mathrm{G}) \alpha_{\mathrm{rs}}\right\}
\end{aligned}
$$

respectively. Where with the above definition for $\mathrm{H}$ (see, equation (36)) the compliance operator is defined as

$$
\begin{aligned}
& \mathrm{Q}_{\mathrm{klij}}=\frac{-\partial^{2} \mathrm{H}}{\partial \alpha_{\mathrm{kl}} \partial \alpha_{\mathrm{ij}}} \\
& \mathrm{Q}_{\mathrm{klij}}=\operatorname{Am} \mathrm{g}_{2}^{\mathrm{m}-1}\left[\frac{(\mathrm{m}-1)}{\mathscr{d}_{2}} \alpha_{\mathrm{kl}} \alpha_{\mathrm{ij}}+\mathrm{I}_{\mathrm{ijkl}}\right]
\end{aligned}
$$

with

$$
\mathrm{I}_{\mathrm{ijk} \mathbf{l}}=\delta_{\mathrm{ik}} \delta_{\mathrm{jl}}
$$

Thus considering the case when $m=1$, the classically (i.e., first term below) assumed linear kinematic evolutionary law [8] can be recovered, i.e.,

$$
\dot{\alpha}_{\mathrm{ij}}=\hat{\mathrm{A}}\left\{\dot{\epsilon}_{\mathrm{ij}}^{\mathrm{I}}-\frac{\mathrm{R}}{\mathrm{H} \kappa^{2}} \mathrm{~g}(\mathrm{G}) \alpha_{\mathrm{ij}}\right\}
$$

in which the evolution of back stress is proportional to the evolution of the irreversible strain rate minus a thermal recovery term. Clearly, this analytical form allows for steady state (i.e., $\dot{\alpha}_{\mathrm{ij}}=0$ ) without the added requirement that the inelastic strain be zero.

Considering the more general case given above, i.e., $m>1$, the commonly assumed evolution equation for the back stress $\left(\alpha_{\mathrm{ij}}\right)$ containing a strain induced recovery term can be recovered under a multiaxial state of stress. However, as opposed to the conventional approach in which an arbitrary function [42] is multiplied by $\left\|\dot{\epsilon}_{\mathrm{ij}} \mathrm{I}_{\mathrm{j}}\right\|$, the present form of the thermodynamically based derivation is seen to lead to the inclusion of a specific scaling 
factor $\eta$ (i.e., the projected back stress length) for that same term (see equations below). This can be seen by substituting in the values (cf. equation(49)) for the compliance operator into equation (48), that is,

$$
\dot{\alpha}_{\mathrm{ij}}=\frac{1}{\mathrm{q}}\left[\frac{(\mathrm{m}-1)}{\not 2} \alpha_{\mathrm{kl}} \alpha_{\mathrm{ij}}+\mathrm{I}_{\mathrm{klij}}\right]^{-1}\left\{\dot{\epsilon}_{\mathrm{kl}}^{\mathrm{I}}-\frac{\mathrm{R}}{\mathrm{H} \kappa^{2}} \mathrm{~g}(\mathrm{G}) \alpha_{\mathrm{kl}}\right\}
$$

where $\quad \mathrm{q}=\mathrm{A} \mathrm{m} \mathrm{g}_{2}^{\mathrm{m}-1}$

An important and not trivial next step, is the inversion [32,42] of the compliance operator. This inversion may not always be available in analytical form, although in the above case it is and equation (51) becomes,

$$
\dot{\alpha}_{\mathrm{ij}}=\frac{1}{\mathrm{q}}\left[\mathrm{I}_{\mathrm{klij}}-\frac{\hat{\mathrm{m}}}{\delta_{2}} \alpha_{\mathrm{kl}} \alpha_{\mathrm{ij}}\right]\left\{\dot{\epsilon}_{\mathrm{kl}}^{\mathrm{I}}-\frac{\mathrm{R}}{\mathrm{H} \kappa^{2}} \mathrm{~g}(\mathrm{G}) \alpha_{\mathrm{kl}}\right\}
$$

where

$$
\hat{\mathrm{m}}=\frac{(\mathrm{m}-1)}{(2 \mathrm{~m}-1)}
$$

Realizing that

$$
\dot{\epsilon}_{\mathrm{kl}}^{\mathrm{I}}=\mathrm{n}_{\mathrm{kl}}\left\|\dot{\epsilon}_{\mathrm{kl}}^{\mathrm{I}}\right\|
$$

it is easily shown that

$$
\dot{\alpha}_{\mathrm{ij}}=\frac{1}{\mathrm{q}}\left[\dot{\epsilon}_{\mathrm{ij}}^{\mathrm{I}}-\eta \alpha_{\mathrm{ij}}\left\|\dot{\epsilon}_{\mathrm{kl}}^{\mathrm{I}}\right\|-\frac{\mathrm{R}}{\mathrm{H} \kappa} 2(1-2 \hat{\mathrm{m}}) \mathrm{g}(\mathrm{G}) \alpha_{\mathrm{ij}}\right]
$$

where

$$
\eta=\frac{\hat{\mathrm{m}}}{g_{2}} \mathrm{n}_{\mathrm{kl}} \alpha_{\mathrm{kl}}
$$

Therefore the fully thermodynamically consistent evolutionary law associated with a complementary dissipation potential whose functional dependence is taken as in equation (36) is equation (53).

Consequently, comparing equation (53) with similar forms found in the literature $[8,10,17,18,19]$ one can see a difference in the leading term typically taken as a constant and here shown to be a function of the back stress, i.e., (1/q) and the lack of an appropriate scaling factor in front of the strain induced recovery term, i.e., $\alpha_{i j}\left\|\dot{\epsilon}_{i j}\right\|$. The 
implications of this scaling factor $\eta$ on the response prediction due to multiaxial states of stress is anticipated to be significant but still remains to be investigated. These differences are related to the fact that here the flow and evolutionary laws are associative and directly linked to the Gibb's potential, through the compliance operator. Another distinction is the resulting desirable numerical features, for example the symmetry in the consistent tangent stiffness matrix produced by the present form, equation (52), and discussed at length in [32]. The disappearance of the strain induced recovery term under a uniaxial state of stress, thus implying no detraction from the uniaxial predictive capability of the model, can be viewed as a price one pays for this symmetry. Inclusion, however, of both a uniaxial as well as multiaxial strain induced recovery term can be achieved by a modification of the assumed form of the present dissipation potential $[8,38]$, thereby suggesting a theory with not only the desired uniaxial predictive capability but all of the theoretical and numerical niceties discussed above as well.

Finally, examining the form proposed by Robinson $[15,16]$, it is clear that this form is merely a truncation of the strain induced recovery term discussed above. Thus, this form as well can easily be modified to be consistent with a complete potential framework by the multiplication of a fourth order tensor (i.e., the inverse of the compliance operator), that is

$$
\dot{\alpha}_{\mathrm{ij}}=\frac{1}{\mathrm{q}} \mathrm{N}_{\mathrm{ijkl}}\left\{\dot{\epsilon}_{\mathrm{kl}}^{\mathrm{I}}-\frac{\mathrm{R}}{\mathrm{H} \kappa^{2}} \mathrm{~g}(\mathrm{G}) \alpha_{\mathrm{ij}}\right\}
$$

where

$$
\mathrm{N}_{\mathrm{ijkl}}=\left[\mathrm{I}_{\mathrm{ijkl}}-\frac{\hat{\mathrm{m}}}{\mathrm{g}_{2}} \alpha_{\mathrm{kl}} \alpha_{\mathrm{ij}}\right]
$$

without altering any of its uniaxial predictive capabilities.

\subsection{CONCLUSION}

A complete potential based framework utilizing internal state variables has been presented for the derivation of reversible and irreversible constitutive equations. This framework assumes the existence of the total (integrated) form of either the (Helmholtz) free energy or the (Gibbs) complementary free energy a priori. Two options for describing the flow and evolutionary equations have been discussed; wherein the fully coupled option the irreversible strain rate is intimately linked to the thermodynamic internal state, hence its functional dependence is completely defined once the free energy is assumed. This 
option was shown to be over restrictive. The second option, the decoupled form, provides significant flexibility since the inelastic strain is taken as an independent state parameter. Here the definition of the inelastic strain rate requires the identification of a complementary dissipation potential. As a consequence of the decoupled form a new operator, i.e. the Compliance operator, is defined which provides a link between the assumed Gibb's and complementary dissipation potential and ensures a number of desirable theoretical and numerical features. It has been shown that although many theories in the literature do not conform to the general potential framework outlined, it is still possible in some cases, by slight modifications of the employed forms, to restore the complete potential structure.

\section{ACKNOWLEDGEMENT:}

This work was initiated during the first author's tenure at ONERA, while participating in a NASA/ONERA exchange program. This author is grateful to Dr. Jean-Louis Chaboche for a number of stimulating discussions on the subject of thermodynamics and viscoplasticity. This together with the concurrent computational investigation in [32] resulted in a revisiting of the first author's thesis (see,[40]) work and consequently this paper.

\section{REFERENCES:}

1) Coleman, B. D., and Gurtin, M. E., "Thermodynamics with Internal State Variables," J. Chem. Phys., Vol. 47, No. 2, 1967, pp. 597-613.

2) Lubliner, J., "On the Structure of Rate Equations of Materials with Internal Variables," Acta Mechanica, Vol. 17, 1973, pp. 109-119.

3) Lubliner, J., "On the Thermodynamic Foundations of Nonlinear Solid Mechanics," Int. J. Nonlinear Mech., Vol. 7, 1972, pp. 237-254.

4) Lehmann, T. H., "Thermodynamical Foundations of Large Inelastic Deformations of Solid Bodies including Damage," Int. J. Plasticity, Vol. 7, 1991, pp. 79-98.

5) Germain, P., Nguyen, Q.S., and Suquet, P., "Continuum Thermodynamics," J. Appl. Mech., ASME, Vol. 50, 1983, pp. 1010-1020.

6) Onat, E.T., and Leckie, F.A., "Representation of Mechanical Behavior in the Presence of Changing Internal Structure," J. Appl. Mech., ASME, Vol. 55, 1988 , pp. $1-10$.

7) Rice, J. R.,"Inelastic Constitutive Relations for Solids: An Internal-Variable Theory and Its Application to Metal Plasticity," J. Mech. Phys, Solids, Vol. 19., 1971, p. 433.

8) Lemaitre, J. and Chaboche, J.L., Mechanics of Solid Materials, Cambrige Univ. Press, N.Y., 1990. 
9) Rice, J.R., "On the Structure of Stress-Strain Relations for Time-Dependent Plastic Deformation in Metals," J. Appl. Mech., ASME, Vol. 37, 1970, pp. 728-

10) Miller, A.K., ed., Unified Constitutive Equations for Plastic Deformation and Creep of Engineering Alloys, Elsevier Applied Science, New York, 1987.

11) Martin, J.B., Plasticity: Fundamentals and General Results, MIT Press, Cambridge, 1975.

12) Kachanov, L.M., Introduction to Continuum Damage Mechanics, Martinus Nijhoff, The Netherlands, 1986.

13) Krajcinovic, D., "Damage Mechanics," Mech. of Mat., Vol 8, 1989, pp. $117-197$.

14) Krajcinovic, D., "Constitutive Equations for Damaging Materials," J. Appl. Mech., ASME, Vol. 50, 1983, pp. 355-360.

15) Robinson, D.N., Duffy, S.F., and Ellis, J.R., " A Viscoplastic Constitutive Theory for Metal Matrix Composites at High Temperature," in Thermo-mechanical Fatigue, H. Sehitoglu and S. Y. Zamrik (eds.), Vol 123, Pressure Vessels and Piping Div., ASME, NY, 1987, pp. 49-56.

16) Robinson, D.N., and Duffy, S.F., "Continuum Deformation Theory for High Temperature Metallic Composites," J. Eng. Mech., ASCE, Vol 16., No. 4, 1990, pp. 832-844.

17) Freed, A.D., Chaboche, J.L., and Walker, K.P., "A Viscoplastic Theory with Thermodynamic Considerations," Acta Mech, to appear, 1991.

18) Chaboche, J.L., "Viscoplastic Constitutive Equations for the Description of Cyclic and Anisotropic Behavior of Metals," Bull. Acad. Pol.Sci., Ser.Sci. Tech., Vol. 25, 1977, pp. 33-39.

19) Miller, A., "An Inelastic Constitutive Model for Monotonic, Cyclic, and Creep Deformations: Part I - Equations Development and Analytical Procedures, "J. Eng. Mater. Technol., Vol. 98, 1976, pp.97 - .

20) Ponter, A.R.S. and Leckie, F.A., "Constitutive Relations for the Time-Dependent Deformation of Metals," J. Eng. Mater. Technol., Vol. 98, 1976, pp. $47-51$.

21) Leckie, F. A., "The Micro and Macromechanics of Creep Rupture," Eng. Fract. Mech., Vol. 25, 1986, pp. 505-521.

22) Ponter, A.R.S., "Convexity and Associated Continuum Properties of A Class of Constitutive Relationships," European J. Mech.,A. Solids, Vol. 15, No. 4, 1976, pp. 527-542.

23) Hodge, P.G., "A Deformation Bounding Theorem for Flow-Law Plasticity," Quart. Appl. Math., Vol. 24, 1966, pp. 171-178. 
24) Carter, P., and Martin, J.B., "Work Bounding Functions for Plastic Materials," J. Appl. Mech., ASME, Vol. 98, 1976, pp. 434-441.

25) Ponter, A. R. S., "General Theorems for the Dynamic Loading of Structures for a State Variable Description of Material Behavior," Inst. of Physics, Conf. Series, No. 47, Ch. 1, 1979, pp. 130-141.

26) Ponter, A.R.S., " Dynamic Behavior of Structures Composed of Strain and Work Hardening Viscoplastic Materials," Int. J. Solids Struct., Vol. 16, 1980, pp. 793-806.

27) Eshelby, J.B.,"The Continuum Theory of Lattice Defects," Solid State Physics, F. Seitz and D. Turnbull (eds.), Vol. 3, Academic Press, N.Y., 1956.

28) Eshelby, J.B., " The force on an Elastic Singularity," Phil. Trans. Roy. Soc., London, Vol. 87, 1951, pp. 12-111.

29) Rice, J.R., "A Path-Independent Integral and the Approximate Analysis of Strain Concentrations by Notches and Cracks," J. Appl. Mech., ASME, Vol. 35, 1968, pp. 379-386.

30) Martin, J.B., Reddy, B.D., Griffin, T.B., and Bird, W.W.,"Applications fo Mathematical Programming Concepts to Incremental Elastic-Plastic Analysis," Eng. Struct., Vol. 9, 1987, pp. 171-176.

31) Cohn, M.Z. and Maier, G. (eds.), Engineering Plasticity by Mathematical Programming, Pergamon Press, Oxford, 1979.

32) Saleeb, A.F., Wilt, T. and Chang, T.Y., "Coupled Viscoplastic - Damage Analysis of Anisotropic Composite Laminates: Computational Aspects and Algorithmic Developments," preprint, 1991.

33) Ortiz, M., and Popv, E. P., "Accuracy and Stability of Integration Algorithms for Elastoplastic Constitutive Equations," Int. J. Num. Meth. Eng., Vol. 21, 1985, pp. 1561-1576.

34) Maier, G. and Novati, g. , " Extremum Theorems for Finite Step Backward Difference Analysis of Elastic Plastic Nonlinearly Hardening Solids," Int. J. Plasticity, Vol. 6, 1990, pp. 1-10.

35) Saleeb, A.F., Chang, T.Y., Graf, W., and Yingyeunyong, "A Hybrid/Mixed Model For Nonlinear Shell Analysis and Its Applications To Large Rotation Problems," Int. J. Num. Meth. Eng., 1988,

36) Arnold, S.M. and Tan, H.Q., "Symbolic Derivation of Potential Based Constitutive Equations," Computational Mechanics, Vol. 6, 1990, pp.237-246.

37) Arnold, S.M., Tan, H.Q. and Dong, X., "Application of Symbolic Computations to the Constitutive Modeling of Structural Materials," in Symbolic Computations and Their Impact on Mechanics, eds. A.K. Noor, I. Elishakoff, and G. Hulbert, ASME, PVP - Vol. 205, 1990, pp. 215-229. 
38) Arnold, S.M. and Saleeb, A.F., "A Complete Potential Based Viscoplastic Model Including Kinematic and Isotropic Hardening," preprint, 1991.

39) Malvern, L.E., Introduction to the Mechanics of a Continuous Medium, Prentice Hall, 1969.

40) Arnold, S.M., "Effects of State Recovery On Creep Buckling Induced By Thermomechanical Loading," PhD. dissertation, University of Akron, Akron, $\mathrm{OH}, 1987$.

41) Chen W.F. and Saleeb A.F., Constitutive Equations for Engineering Materials, Volume I: Elasticity and Modeling, John Wiley \& Sons, 1982.

42) Saleeb, A.F., and Arnold, S.M., "Unified Viscoplastic Models: Some Currently-Üsed Mathematical Forms, Possible Modifications, and Their Underlying Potential Structure," preprint, 1991. 

- 
Public reporting burden for this collection of information is estimaled to average 1 hour per response, including the time for reviewing instructions, searching existing data sources, gathering and maintaining the data needed, and completing and reviewing the collection of information. Send comments regarding this burden estimate or any olher aspect of this collection of information, including suggestions for reducing this burden, to Washington Headquarters Services, Directorate for intormation Operations and Reports, 1215 Jefferson Davis Highway, Suite 1204, Arlington, VA 22202-4302, and to the Office of Management and Budget, Paperwork Reduction Project (0704-0188), Washington, DC 20503.

\begin{tabular}{|l|c|c|}
\hline 1. AGENCY USE ONLY (Leave blank) & $\begin{array}{c}\text { 2. REPORT DATE } \\
\text { November } 1991\end{array}$ & $\begin{array}{r}\text { 3. REPORT TYPE AND DATES COVERED } \\
\text { Technical Memorandum }\end{array}$ \\
\hline
\end{tabular}

\section{TITLE AND SUBTITLE}

5. FUNDING NUMBERS

On the Thermodynamic Framework of Generalized Coupled

Thermoelastic-Viscoplastic-Damage Modeling

\section{AUTHOR(S)}

WU $-510-01-50$

S.M. Arnold and A.F. Saleeb

7. PERFORMING ORGANIZATION NAME(S) AND ADDRESS(ES)

National Aeronautics and Space Administration

Lewis Research Center

Cleveland, Ohio 44135-3191

8. PERFORMING ORGANIZATION

REPORT NUMBER

E-6723

9. SPONSORING/MONITORING AGENCY NAMES(S) AND ADDRESS(ES)

10. SPONSORING/MONITORING AGENCY REPORT NUMBER

National Aeronautics and Space Administration

Washington, D.C. 20546-0001

NASA TM -105349

11. SUPPLEMENTARY NOTES

S.M. Arnold, NASA Lewis Research Center; A.F. Saleeb, University of Akron, Department of Civil Engineering, Akron, Ohio 44325. Responsible person, S.M. Arnold, (216) 433-3334.

12a. DISTRIBUTION/AVAILABILITY STATEMENT

12b. DISTRIBUTION CODE

Unclassified - Unlimited

Subject Category 39

13. ABSTRACT (Maximum 200 words)

A complete potential based framework utilizing internal state variables is put forth for the derivation of reversible and irreversible constitutive equations. In this framework the existence of the total (integrated) form of either the (Helmholtz) free energy or the (Gibbs) complementary free energy are assumed a priori. Two options for describing the flow and evolutionary equations are described, wherein option one (the fully coupled form) is shown to be over restrictive while the second option (the decoupled form) provides significant flexibility. As a consequence of the decoupled form a new operator, i.e., the Compliance operator, is defined which provides a link between the assumed Gibb's and complementary dissipation potential and ensures a number of desirable numerical features, for example the symmetry of the resulting consistent tangent stiffness matrix. An important conclusion reached, is that although many theories in the literature do not conform to the general potential framework outlined, it is still possible in some cases, by slight modifications of the employed forms, to restore the complete potential structure.

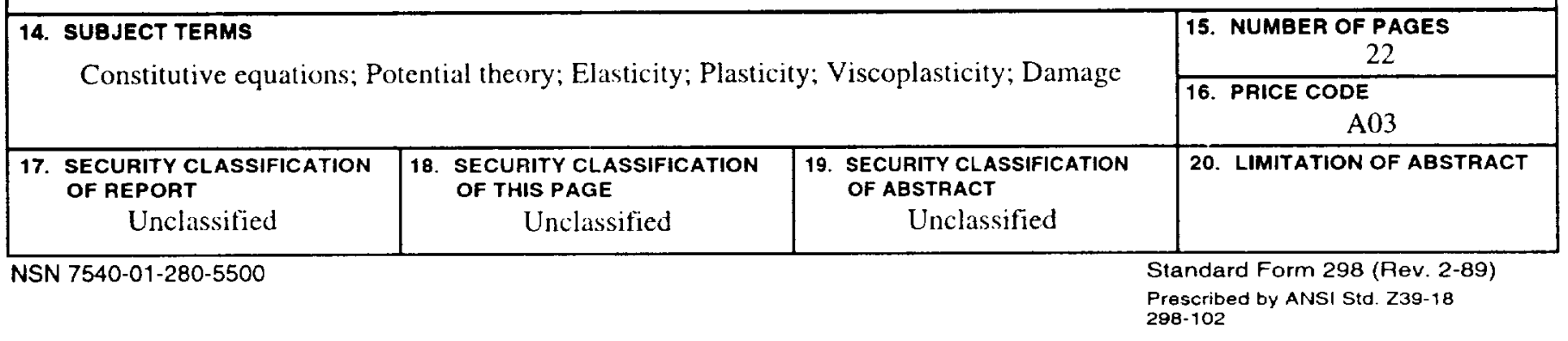




\author{
$-$
}

